\title{
Pneumocystis jirovecii pneumonia prophylaxis in a 42-year-old woman on immunosuppressive therapy
}

\author{
Sheliza Halani MD, Nisha Andany MD MPH, Rupal Shah MD MHPE
}

Cite as: CMAJ 2020 October 26;192:E1306-8. doi: 10.1503/cmaj.200846

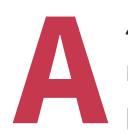

42-year-old woman, with a 2-year history of dermatomyositis controlled with prednsione (5 mg once daily), presents to her family physician 1 week after a flare that required admission to hospital. The patient's symptoms include proximal muscle weakness and dyspnea secondary to interstitial lung disease. High-dose prednisone $(60 \mathrm{mg}$ orally daily) is started, with plans for prolonged taper. Her family physician questions whether she needs prophylaxis for Pneumocystis jirovecii pneumonia.

\section{Is this patient at risk of Pneumocystis jirovecii pneumonia?}

Pneumocystis jirovecii pneumonia is an opportunistic fungal infection. ${ }^{1,2}$ Impaired cell-mediated immunity is the predominant risk factor; a link best established in patients with HIV and CD4 counts of less than 200 cells $/ \mathrm{mm}^{3} .{ }^{3}$ However, increasing rates of Pneumocystis jirovecii pneumonia in patients without HIV (Box 1) highlight the role of non-HIV-mediated immunosuppression. ${ }^{1-5}$ Glucocorticoid therapy impairs cell-mediated immunity by reducing CD4 lymphocytes and attenuating signalling in inflammatory cascades. ${ }^{3,6}$ Although corticosteroids confer the greatest risk when combined with a high-risk underlying condition (i.e., hematologic malignant disease) or additional immunosuppressive agents, monotherapy has also been associated with Pneumocystis jirovecii pneumonia. ${ }^{3}$ In a prospective cohort study involving patients with rheumatologic diseases who were taking prednisone ( $\geq 30 \mathrm{mg}$ daily for at least $4 \mathrm{wk}$ ), 1-year incidence of Pneumocystis jirovecii pneumonia was 2.37 cases per 100 person-years. ${ }^{7}$ Higher doses of prednisone ( $\geq 60 \mathrm{mg}$ ), concomitant cyclophosphamide pulse therapy, increased age and baseline lymphopenia exacerbated this risk. ${ }^{7}$ A retrospective analysis involving patients without HIV found that Pneumocystis jirovecii pneumonia can occur at corticosteroid doses as low as $16 \mathrm{mg} /$ day and durations of 8 weeks or less. ${ }^{3}$ This patient's history of dermatomyositis and recent flare requiring high-dose prednisone increases her risk of Pneumocystis jirovecii pneumonia.

\section{How would Pneumocystis jirovecii pneumonia present clinically in this patient?}

Although clinical presentations may be varied and nonspecific, typical symptoms in this patient would include acute dyspnea, cough and fever over days. ${ }^{8}$ This contrasts with the indolent onset of symptoms over several days to weeks observed in those with HIV. ${ }^{8}$ A 2014 prospective study in France reported that patients with Pneumocystis jirovecii pneumonia who were HIV negative had fewer reported symptoms at time of presentation (contributing to delays in diagnosis), were started on treatment later, and had higher rates of mechanical ventilation and death than patients who were HIV positive. ${ }^{8}$

Clinicians should maintain a high index of suspicion for Pneumocystis jirovecii pneumonia in patients with non-HIV-mediated immunosuppression, as diagnostic confirmation can be challenging. Surrogate markers are not useful; tests for lactate dehydrogenase levels have $63 \%$ sensitivity in patients who are HIV negative compared with greater than $90 \%$ in those who are HIV positive. ${ }^{9}$ Although computed tomography of the chest will show bilateral ground-glass opacities in both populations, diagnostic confirmation requires direct visualization of the organism. ${ }^{4}$ Detection methods, such as bronchoalveolar lavage, have lower sensitivity in patients who are HIV negative owing to a lower burden of organisms. ${ }^{4}$

\section{Should this patient receive prophylaxis for Pneumocystis jirovecii pneumonia?}

The decision to start prophylaxis in this patient is nuanced, warranting careful consideration of benefits and risks; however, it is supported by evidence. ${ }^{7}$ Prophylaxis is recommended when the risk of Pneumocystis jirovecii pneumonia is greater than $3.5 \% .^{5}$ The strongest evidence for prophylaxis in HIV-negative populations exists for solid organ or hematopoietic stem cell transplantation, solid tumours on T-cell-depleting agents, hematologic malignant disease, primary immune deficiencies, vasculitides and autoimmune conditions treated with tumour-necrosis-factor- $\alpha$ inhibitors (Box 1). 1,2,4,5 
Box 1: Indications for prophylaxis for Pneumocystis jirovecii pneumonia in patients who are immunocompromised and HIV negative

\section{Patient \\ population}

Solid organ transplantation

Hematopoietic stem cell transplantation malignant disease

Autoimmune diseases and vasculitis

Primary immune deficiency

Glucocorticoid therapy (other) $\dagger$
Solid tumour

For 6-12 mo after transplantation*

Autologous stem cell transplant

- For 3-6 mo

Allogeneic stem cell transplant

- For at least $6 \mathrm{mo}$

- Longer duration may be required with chronic GVHD or ongoing immunosuppressive therapy

Glucocorticoids $\uparrow$ equivalent to prednisone $\geq 20 \mathrm{mg} / \mathrm{d}$ for $\geq 4 \mathrm{wk}$

Acute lymphoblastic leukemia receiving chemotherapy

Alemtuzumab

- During therapy and for at least $2 \mathrm{mo}$ after cessation (and until CD4 count $>200$ cells $/ \mathrm{mm}^{3}$ )

T-cell depleting agents (e.g., fludaribine or cladribine)

- Until CD4 count $>200$ cells $/ \mathrm{mm}^{3}$

Glucocorticoids $\uparrow$ equivalent to prednisone $\geq 20 \mathrm{mg} / \mathrm{d}$ for $4 \mathrm{wk}$

Temozolomide and radiation therapy

- Until CD4 count $>200$ cells $/ \mathrm{mm}^{3}$

Rheumatoid arthritis receiving TNF- $\alpha$ inhibitors

- If also receiving glucocorticoids $†$ or other immunosuppressive agents

Inflammatory bowel disease receiving TNF- $\alpha$ inhibitors

- If also receiving glucocorticoids $†$ or other immunosuppressive agents

Granulomatous polyangiitis receiving cyclophosphamide

Vasculitis associated with ANCA

- Receiving cyclophosphamide and glucocorticoids $\dagger$

Other vasculitis

- Receiving glucocorticoids $†$ and a 2nd immunosuppressive agent

Connective tissue disease

- Receiving glucocorticoids $\dagger$ equivalent to prednisone $\geq 20 \mathrm{mg} / \mathrm{d}$ for $\geq 4 \mathrm{wk}$

Severe combined immune deficiency Idiopathic CD4 lymphocytopenia $X$-linked hyper-IgM syndrome

Prednisone equivalent $\geq 20 \mathrm{mg} / \mathrm{d}$ for $\geq 4$ wk plus one of:

- Additional immunosuppressive agents

- Steroid-sparing antirheumatic drugs

- Underlying immunosuppressive condition

\section{Other populations (where prophylaxis may be considered)}

Glucocorticoid

therapy†

Prednisone equivalent $\geq 20 \mathrm{mg} / \mathrm{d}$ for $\geq 4 \mathrm{wk}$ - With or without underlying lung disease

Note: $\mathrm{ANCA}=$ antineutrophil cytoplasmic antibody, GVHD = graft versus host disease, IgM = immunoglobulin M, TNF = tumour necrosis factor.

*Duration varies with organ transplanted and requirement for other immunosuppressive agents (kidney transplant recipients may receive a shorter duration of prophylaxis).

†Glucocorticoids (prednisone equivalent of $\geq 20 \mathrm{mg} / \mathrm{d}$ for $\geq 4$ wk; some experts suggest $\geq 16-20 \mathrm{mg} / \mathrm{d})$
Two meta-analyses that compared prophylaxis using trimethoprim-sulfamethoxazole (TMP-SMX) with placebo or no prophylaxis in high-risk patients with hematologic malignant disease or transplantation reported numbers-needed-to-treat (NNT) as low as 15 and 19 to prevent 1 case of Pneumocystis jirovecii pneumonia; the overall rate of adverse events was $3.1 \%$, with no difference between groups., ${ }^{1,5}$

Among connective tissue diseases, incidence of Pneumocystis jirovecii pneumonia is highest in the presence of granulomatosis with polyangiitis ( $8 \%-12 \%)$, polyarteritis nodosa $(6.5 \%)$ and dermatomyositis/polymyositis (2.7\%). ${ }^{4}$ Although the isolated risk of Pneumocystis jirovecii pneumonia with inflammatory myopathies falls below 3.5\%, these conditions are associated with T-cell lymphopenia ${ }^{10}$ and often require glucocorticoid therapy; concomitant interstitial lung disease exacerbates this risk. ${ }^{11}$ In a cohort study involving 1092 patients with rheumatologic diseases who were taking moderate- to high-dose steroids, some patients were treated with TMP-SMX prophylaxis according to the judgment of the treating physicians. ${ }^{7}$ Prophylaxis with TMP-SMX reduced 1-year incidence of Pneumocystis jirovecii pneumonia by $93 \%$ and associated mortality by $92 \% .^{7}$ The NNT to prevent 1 case of Pneumocystis jirovecii pneumonia was 52; serious adverse drug reactions were uncommon (number-needed-to-harm $=131$ ). ${ }^{7}$ In the absence of consensus guidelines, existing literature favours prophylaxis in this patient with dermatomyositis and interstitial lung disease that requires prolonged high-dose steroids.

\section{What are the options for Pneumocystis jirovecii pneumonia prophylaxis?}

The preferred agent is TMP-SMX. ${ }^{2}$ Dosing regimens include 1 doublestrength tablet 3 times per week or 1 single-strength tablet daily, with no difference in efficacy or toxicity. ${ }^{1}$ Adverse reactions include rash, myelosuppression, and liver and renal toxicities. ${ }^{1,7}$ Alternative options include dapsone, atovaquone and aerosolized pentamidine based on limited evidence and largely extrapolated from the HIV literature. ${ }^{2,4}$

\section{Case revisited}

This patient has multiple risk factors for Pneumocystis jirovecii pneumonia, including underlying rheumatologic disease that requires long-term, high-dose glucocorticoids. She is at risk of severe infection and poor outcomes if Pneumocystis jirovecii pneumonia develops, particularly with concurrent interstitial lung disease. Based on available evidence, the benefits of prophylaxis outweigh the risks of harm. In consultation with rheumatology, she started prophylaxis with TMP-SMX and monitoring for adverse events.

\section{References}

1. Stern A, Green H, Paul M, et al. Prophylaxis for Pneumocystis pneumonia (PCP) in non-HIV immunocompromised patients. Cochrane Database Syst Rev 2014;2014:CD005590.

2. Carmona EM, Limper AH. Update on diagnosis and treatment of Pneumocystis pneumonia. Ther Adv Respir Dis 2011;5:41-59.

3. Yale SH, Limper AH. Pneumocystis carinii pneumonia in patients without acquired immunodeficiency syndrome: associated illnesses and prior corticosteroid therapy. Mayo Clin Proc 1996;71:5-13. 
4. Roux A, Gonzalez F, Roux M, et al. Groupe de recherche respiratoire en réanimation en onco-hématologie (Grrr-OH). Update on pulmonary Pneumocystis jirovecii infection in non-HIV patients. Med Mal Infect 2014;44:185-98.

5. Green H, Paul M, Vidal L, et al. Prophylaxis of Pneumocystis pneumonia in immunocompromised non-HIV-infected patients: systematic review and metaanalysis of randomized controlled trials. Mayo Clin Proc 2007;82:1052-9.

6. Cain DW, Cidlowski JA. Immune regulation by glucocorticoids. Nat Rev Immunol 2017;17:233-47.

7. Park JW, Curtis JR, Moon J, et al. Prophylactic effect of trimethoprimsulfamethoxazole for Pneumocystis pneumonia in patients with rheumatic diseases exposed to prolonged high-dose glucocorticoids. Ann Rheum Dis 2018;77:644-9.

8. Roux A, Canet E, Valade S, et al. Pneumocystis jirovecii pneumonia in patients with or without AIDS, France. Emerg Infect Dis 2014;20:1490-7.

9. Vogel M, Weissgerber P, Goeppert B, et al. Accuracy of serum LDH elevation for the diagnosis of Pneumocystis jirovecii pneumonia. Swiss Med Wkly 2011;141:w13184.

10. Zhang L, Xia Q, Li W, et al. The RIG-I pathway is involved in peripheral T-cell lymphopenia in patients with dermatomyositis. Arthritis Res Ther 2019;21:131.

11. Kadoya A, Okada J, likuni Y, et al. Risk factors for Pneumocystis carinii pneumonia in patients with polymyositis/dermatomyositis or systemic lupus erythematosus. J Rheumatol 1996;23:1186-8.
Competing interests: Nisha Andany reports participation as a site investigator for HIV clinical trials sponsored by Gilead Sciences, GlaxoSmithKline and Janssen (outside of submitted work). No other competing interests were declared.

This article has been peer reviewed.

The clinical scenario is fictional.

Affiliations: Department of Medicine (Halani, Andany, Shah), University of Toronto; Division of Infectious Diseases (Andany), Sunnybrook Health Sciences Centre; Division of General Internal Medicine (Shah), Toronto Western Hospital, Toronto, Ont.

Contributors: All of the authors contributed to the conception, design and drafting of the work, gave final approval of the version to be published and agreed to be accountable for all aspects of the work.

Correspondence to: Rupal Shah, rupal.shah@uhn.ca 